\title{
Algebraic Logic Perspective on Prucnal's Substitution
}

\author{
Alex Citkin
}

\begin{abstract}
A term $\operatorname{td}(p, q, r)$ is called a ternary deductive (TD) term for a variety of algebras $\mathcal{V}$ if the identity $t d(p, p, r) \approx r$ holds in $\mathcal{V}$ and $(\mathrm{c}, \mathrm{d}) \in \theta(\mathrm{a}, \mathrm{b})$ yields $t d(\mathrm{a}, \mathrm{b}, \mathrm{c}) \approx t d(\mathrm{a}, \mathrm{b}, \mathrm{d})$ for any $\mathcal{A} \in \mathcal{V}$ and any principal congruence $\theta$ on $\mathcal{A}$. A connective $f\left(p_{1}, \ldots, p_{n}\right)$ is called $t d$-distributive if $t d\left(p, q, f\left(r_{1}, \ldots, r_{n}\right)\right) \approx f\left(t d\left(p, q, r_{1}\right), \ldots, t d\left(p, q, r_{n}\right)\right)$. If $\mathrm{L}$ is a propositional logic and $\mathcal{V}$ is a corresponding variety (algebraic semantic) that has a $\mathrm{TD}$ term $t d$, then any admissible in $\mathrm{L}$ rule, the premises of which contain only $t d$-distributive operations, is derivable, and the substitution $r \mapsto t d(p, q, r)$ is a projective L-unifier for any formula containing only $t d$-distributive connectives. The above substitution is a generalization of the substitution introduced by T. Prucnal to prove structural completeness of the implication fragment of intuitionistic propositional logic.
\end{abstract}

\section{Introduction}

In this paper, we study admissibility of structural inference rules in algebraizable (propositional) logics. A (structural inference) rule is an expression of the form $A_{1}, \ldots, A_{n} / B$, where $A_{1}, \ldots, A_{n}$ are (propositional) formulas called premises (of the rule) and $B$ is a formula called the conclusion (of the rule). We recall that given a (propositional) logic $\mathrm{L}$, a rule $A_{1}, \ldots, A_{n} / B$ is admissible in $\mathrm{L}$ if the logic $\mathrm{L}$ is closed under this rule; that is, for any substitution $\sigma$ (of formulas for propositional variables), formula $\sigma(B)$ is valid in $L$ as long as all formulas $\sigma\left(A_{1}\right), \ldots, \sigma\left(A_{n}\right)$ are valid in $\mathrm{L}$. A substitution that simultaneously makes all formulas $A_{1}, \ldots, A_{n}$ valid in $\mathrm{L}$ is known as an L-unifier of formulas $A_{1}, \ldots, A_{n}$. An L-unifier of a single formula is a substitution that makes this formula valid in $L$. Thus, a rule is admissible in a

Received September 30, 2012; accepted January 30, 2014

First published online August 13, 2016

2010 Mathematics Subject Classification: Primary 03B55, 03B60; Secondary 03G27, 03G25

Keywords: propositional logic, admissible inference rule, unification, hereditary structural completeness, algebraic semantic, Prucnal's substitution, ternary deduction term

(C) 2016 by University of Notre Dame 10.1215/00294527-3659423 


\title{
Algebraic Logic Perspective on Prucnal's Substitution
}

\author{
Alex Citkin
}

\begin{abstract}
A term $\operatorname{td}(p, q, r)$ is called a ternary deductive (TD) term for a variety of algebras $\mathcal{V}$ if the identity $t d(p, p, r) \approx r$ holds in $\mathcal{V}$ and $(\mathrm{c}, \mathrm{d}) \in \theta(\mathrm{a}, \mathrm{b})$ yields $t d(\mathrm{a}, \mathrm{b}, \mathrm{c}) \approx t d(\mathrm{a}, \mathrm{b}, \mathrm{d})$ for any $\mathcal{A} \in \mathcal{V}$ and any principal congruence $\theta$ on $\mathcal{A}$. A connective $f\left(p_{1}, \ldots, p_{n}\right)$ is called $t d$-distributive if $t d\left(p, q, f\left(r_{1}, \ldots, r_{n}\right)\right) \approx f\left(t d\left(p, q, r_{1}\right), \ldots, t d\left(p, q, r_{n}\right)\right)$. If $\mathrm{L}$ is a propositional logic and $\mathcal{V}$ is a corresponding variety (algebraic semantic) that has a $\mathrm{TD}$ term $t d$, then any admissible in $\mathrm{L}$ rule, the premises of which contain only $t d$-distributive operations, is derivable, and the substitution $r \mapsto t d(p, q, r)$ is a projective L-unifier for any formula containing only $t d$-distributive connectives. The above substitution is a generalization of the substitution introduced by T. Prucnal to prove structural completeness of the implication fragment of intuitionistic propositional logic.
\end{abstract}

\section{Introduction}

In this paper, we study admissibility of structural inference rules in algebraizable (propositional) logics. A (structural inference) rule is an expression of the form $A_{1}, \ldots, A_{n} / B$, where $A_{1}, \ldots, A_{n}$ are (propositional) formulas called premises (of the rule) and $B$ is a formula called the conclusion (of the rule). We recall that given a (propositional) logic $\mathrm{L}$, a rule $A_{1}, \ldots, A_{n} / B$ is admissible in $\mathrm{L}$ if the logic $\mathrm{L}$ is closed under this rule; that is, for any substitution $\sigma$ (of formulas for propositional variables), formula $\sigma(B)$ is valid in $L$ as long as all formulas $\sigma\left(A_{1}\right), \ldots, \sigma\left(A_{n}\right)$ are valid in $\mathrm{L}$. A substitution that simultaneously makes all formulas $A_{1}, \ldots, A_{n}$ valid in $\mathrm{L}$ is known as an L-unifier of formulas $A_{1}, \ldots, A_{n}$. An L-unifier of a single formula is a substitution that makes this formula valid in $L$. Thus, a rule is admissible in a

Received September 30, 2012; accepted January 30, 2014

First published online August 13, 2016

2010 Mathematics Subject Classification: Primary 03B55, 03B60; Secondary 03G27, 03G25

Keywords: propositional logic, admissible inference rule, unification, hereditary structural completeness, algebraic semantic, Prucnal's substitution, ternary deduction term

(C) 2016 by University of Notre Dame 10.1215/00294527-3659423 


\title{
Algebraic Logic Perspective on Prucnal's Substitution
}

\author{
Alex Citkin
}

\begin{abstract}
A term $\operatorname{td}(p, q, r)$ is called a ternary deductive (TD) term for a variety of algebras $\mathcal{V}$ if the identity $t d(p, p, r) \approx r$ holds in $\mathcal{V}$ and $(\mathrm{c}, \mathrm{d}) \in \theta(\mathrm{a}, \mathrm{b})$ yields $t d(\mathrm{a}, \mathrm{b}, \mathrm{c}) \approx t d(\mathrm{a}, \mathrm{b}, \mathrm{d})$ for any $\mathcal{A} \in \mathcal{V}$ and any principal congruence $\theta$ on $\mathcal{A}$. A connective $f\left(p_{1}, \ldots, p_{n}\right)$ is called $t d$-distributive if $t d\left(p, q, f\left(r_{1}, \ldots, r_{n}\right)\right) \approx f\left(t d\left(p, q, r_{1}\right), \ldots, t d\left(p, q, r_{n}\right)\right)$. If $\mathrm{L}$ is a propositional logic and $\mathcal{V}$ is a corresponding variety (algebraic semantic) that has a $\mathrm{TD}$ term $t d$, then any admissible in $\mathrm{L}$ rule, the premises of which contain only $t d$-distributive operations, is derivable, and the substitution $r \mapsto t d(p, q, r)$ is a projective L-unifier for any formula containing only $t d$-distributive connectives. The above substitution is a generalization of the substitution introduced by T. Prucnal to prove structural completeness of the implication fragment of intuitionistic propositional logic.
\end{abstract}

\section{Introduction}

In this paper, we study admissibility of structural inference rules in algebraizable (propositional) logics. A (structural inference) rule is an expression of the form $A_{1}, \ldots, A_{n} / B$, where $A_{1}, \ldots, A_{n}$ are (propositional) formulas called premises (of the rule) and $B$ is a formula called the conclusion (of the rule). We recall that given a (propositional) logic $\mathrm{L}$, a rule $A_{1}, \ldots, A_{n} / B$ is admissible in $\mathrm{L}$ if the logic $\mathrm{L}$ is closed under this rule; that is, for any substitution $\sigma$ (of formulas for propositional variables), formula $\sigma(B)$ is valid in $L$ as long as all formulas $\sigma\left(A_{1}\right), \ldots, \sigma\left(A_{n}\right)$ are valid in $\mathrm{L}$. A substitution that simultaneously makes all formulas $A_{1}, \ldots, A_{n}$ valid in $\mathrm{L}$ is known as an L-unifier of formulas $A_{1}, \ldots, A_{n}$. An L-unifier of a single formula is a substitution that makes this formula valid in $L$. Thus, a rule is admissible in a

Received September 30, 2012; accepted January 30, 2014

First published online August 13, 2016

2010 Mathematics Subject Classification: Primary 03B55, 03B60; Secondary 03G27, 03G25

Keywords: propositional logic, admissible inference rule, unification, hereditary structural completeness, algebraic semantic, Prucnal's substitution, ternary deduction term

(C) 2016 by University of Notre Dame 10.1215/00294527-3659423 


\title{
Algebraic Logic Perspective on Prucnal's Substitution
}

\author{
Alex Citkin
}

\begin{abstract}
A term $\operatorname{td}(p, q, r)$ is called a ternary deductive (TD) term for a variety of algebras $\mathcal{V}$ if the identity $t d(p, p, r) \approx r$ holds in $\mathcal{V}$ and $(\mathrm{c}, \mathrm{d}) \in \theta(\mathrm{a}, \mathrm{b})$ yields $t d(\mathrm{a}, \mathrm{b}, \mathrm{c}) \approx t d(\mathrm{a}, \mathrm{b}, \mathrm{d})$ for any $\mathcal{A} \in \mathcal{V}$ and any principal congruence $\theta$ on $\mathcal{A}$. A connective $f\left(p_{1}, \ldots, p_{n}\right)$ is called $t d$-distributive if $t d\left(p, q, f\left(r_{1}, \ldots, r_{n}\right)\right) \approx f\left(t d\left(p, q, r_{1}\right), \ldots, t d\left(p, q, r_{n}\right)\right)$. If $\mathrm{L}$ is a propositional logic and $\mathcal{V}$ is a corresponding variety (algebraic semantic) that has a $\mathrm{TD}$ term $t d$, then any admissible in $\mathrm{L}$ rule, the premises of which contain only $t d$-distributive operations, is derivable, and the substitution $r \mapsto t d(p, q, r)$ is a projective L-unifier for any formula containing only $t d$-distributive connectives. The above substitution is a generalization of the substitution introduced by T. Prucnal to prove structural completeness of the implication fragment of intuitionistic propositional logic.
\end{abstract}

\section{Introduction}

In this paper, we study admissibility of structural inference rules in algebraizable (propositional) logics. A (structural inference) rule is an expression of the form $A_{1}, \ldots, A_{n} / B$, where $A_{1}, \ldots, A_{n}$ are (propositional) formulas called premises (of the rule) and $B$ is a formula called the conclusion (of the rule). We recall that given a (propositional) logic $\mathrm{L}$, a rule $A_{1}, \ldots, A_{n} / B$ is admissible in $\mathrm{L}$ if the logic $\mathrm{L}$ is closed under this rule; that is, for any substitution $\sigma$ (of formulas for propositional variables), formula $\sigma(B)$ is valid in $L$ as long as all formulas $\sigma\left(A_{1}\right), \ldots, \sigma\left(A_{n}\right)$ are valid in $\mathrm{L}$. A substitution that simultaneously makes all formulas $A_{1}, \ldots, A_{n}$ valid in $\mathrm{L}$ is known as an L-unifier of formulas $A_{1}, \ldots, A_{n}$. An L-unifier of a single formula is a substitution that makes this formula valid in $L$. Thus, a rule is admissible in a

Received September 30, 2012; accepted January 30, 2014

First published online August 13, 2016

2010 Mathematics Subject Classification: Primary 03B55, 03B60; Secondary 03G27, 03G25

Keywords: propositional logic, admissible inference rule, unification, hereditary structural completeness, algebraic semantic, Prucnal's substitution, ternary deduction term

(C) 2016 by University of Notre Dame 10.1215/00294527-3659423 


\title{
Algebraic Logic Perspective on Prucnal's Substitution
}

\author{
Alex Citkin
}

\begin{abstract}
A term $\operatorname{td}(p, q, r)$ is called a ternary deductive (TD) term for a variety of algebras $\mathcal{V}$ if the identity $t d(p, p, r) \approx r$ holds in $\mathcal{V}$ and $(\mathrm{c}, \mathrm{d}) \in \theta(\mathrm{a}, \mathrm{b})$ yields $t d(\mathrm{a}, \mathrm{b}, \mathrm{c}) \approx t d(\mathrm{a}, \mathrm{b}, \mathrm{d})$ for any $\mathcal{A} \in \mathcal{V}$ and any principal congruence $\theta$ on $\mathcal{A}$. A connective $f\left(p_{1}, \ldots, p_{n}\right)$ is called $t d$-distributive if $t d\left(p, q, f\left(r_{1}, \ldots, r_{n}\right)\right) \approx f\left(t d\left(p, q, r_{1}\right), \ldots, t d\left(p, q, r_{n}\right)\right)$. If $\mathrm{L}$ is a propositional logic and $\mathcal{V}$ is a corresponding variety (algebraic semantic) that has a $\mathrm{TD}$ term $t d$, then any admissible in $\mathrm{L}$ rule, the premises of which contain only $t d$-distributive operations, is derivable, and the substitution $r \mapsto t d(p, q, r)$ is a projective L-unifier for any formula containing only $t d$-distributive connectives. The above substitution is a generalization of the substitution introduced by T. Prucnal to prove structural completeness of the implication fragment of intuitionistic propositional logic.
\end{abstract}

\section{Introduction}

In this paper, we study admissibility of structural inference rules in algebraizable (propositional) logics. A (structural inference) rule is an expression of the form $A_{1}, \ldots, A_{n} / B$, where $A_{1}, \ldots, A_{n}$ are (propositional) formulas called premises (of the rule) and $B$ is a formula called the conclusion (of the rule). We recall that given a (propositional) logic $\mathrm{L}$, a rule $A_{1}, \ldots, A_{n} / B$ is admissible in $\mathrm{L}$ if the logic $\mathrm{L}$ is closed under this rule; that is, for any substitution $\sigma$ (of formulas for propositional variables), formula $\sigma(B)$ is valid in $L$ as long as all formulas $\sigma\left(A_{1}\right), \ldots, \sigma\left(A_{n}\right)$ are valid in $\mathrm{L}$. A substitution that simultaneously makes all formulas $A_{1}, \ldots, A_{n}$ valid in $\mathrm{L}$ is known as an L-unifier of formulas $A_{1}, \ldots, A_{n}$. An L-unifier of a single formula is a substitution that makes this formula valid in $L$. Thus, a rule is admissible in a

Received September 30, 2012; accepted January 30, 2014

First published online August 13, 2016

2010 Mathematics Subject Classification: Primary 03B55, 03B60; Secondary 03G27, 03G25

Keywords: propositional logic, admissible inference rule, unification, hereditary structural completeness, algebraic semantic, Prucnal's substitution, ternary deduction term

(C) 2016 by University of Notre Dame 10.1215/00294527-3659423 


\title{
Algebraic Logic Perspective on Prucnal's Substitution
}

\author{
Alex Citkin
}

\begin{abstract}
A term $\operatorname{td}(p, q, r)$ is called a ternary deductive (TD) term for a variety of algebras $\mathcal{V}$ if the identity $t d(p, p, r) \approx r$ holds in $\mathcal{V}$ and $(\mathrm{c}, \mathrm{d}) \in \theta(\mathrm{a}, \mathrm{b})$ yields $t d(\mathrm{a}, \mathrm{b}, \mathrm{c}) \approx t d(\mathrm{a}, \mathrm{b}, \mathrm{d})$ for any $\mathcal{A} \in \mathcal{V}$ and any principal congruence $\theta$ on $\mathcal{A}$. A connective $f\left(p_{1}, \ldots, p_{n}\right)$ is called $t d$-distributive if $t d\left(p, q, f\left(r_{1}, \ldots, r_{n}\right)\right) \approx f\left(t d\left(p, q, r_{1}\right), \ldots, t d\left(p, q, r_{n}\right)\right)$. If $\mathrm{L}$ is a propositional logic and $\mathcal{V}$ is a corresponding variety (algebraic semantic) that has a $\mathrm{TD}$ term $t d$, then any admissible in $\mathrm{L}$ rule, the premises of which contain only $t d$-distributive operations, is derivable, and the substitution $r \mapsto t d(p, q, r)$ is a projective L-unifier for any formula containing only $t d$-distributive connectives. The above substitution is a generalization of the substitution introduced by T. Prucnal to prove structural completeness of the implication fragment of intuitionistic propositional logic.
\end{abstract}

\section{Introduction}

In this paper, we study admissibility of structural inference rules in algebraizable (propositional) logics. A (structural inference) rule is an expression of the form $A_{1}, \ldots, A_{n} / B$, where $A_{1}, \ldots, A_{n}$ are (propositional) formulas called premises (of the rule) and $B$ is a formula called the conclusion (of the rule). We recall that given a (propositional) logic $\mathrm{L}$, a rule $A_{1}, \ldots, A_{n} / B$ is admissible in $\mathrm{L}$ if the logic $\mathrm{L}$ is closed under this rule; that is, for any substitution $\sigma$ (of formulas for propositional variables), formula $\sigma(B)$ is valid in $L$ as long as all formulas $\sigma\left(A_{1}\right), \ldots, \sigma\left(A_{n}\right)$ are valid in $\mathrm{L}$. A substitution that simultaneously makes all formulas $A_{1}, \ldots, A_{n}$ valid in $\mathrm{L}$ is known as an L-unifier of formulas $A_{1}, \ldots, A_{n}$. An L-unifier of a single formula is a substitution that makes this formula valid in $L$. Thus, a rule is admissible in a

Received September 30, 2012; accepted January 30, 2014

First published online August 13, 2016

2010 Mathematics Subject Classification: Primary 03B55, 03B60; Secondary 03G27, 03G25

Keywords: propositional logic, admissible inference rule, unification, hereditary structural completeness, algebraic semantic, Prucnal's substitution, ternary deduction term

(C) 2016 by University of Notre Dame 10.1215/00294527-3659423 


\title{
Algebraic Logic Perspective on Prucnal's Substitution
}

\author{
Alex Citkin
}

\begin{abstract}
A term $\operatorname{td}(p, q, r)$ is called a ternary deductive (TD) term for a variety of algebras $\mathcal{V}$ if the identity $t d(p, p, r) \approx r$ holds in $\mathcal{V}$ and $(\mathrm{c}, \mathrm{d}) \in \theta(\mathrm{a}, \mathrm{b})$ yields $t d(\mathrm{a}, \mathrm{b}, \mathrm{c}) \approx t d(\mathrm{a}, \mathrm{b}, \mathrm{d})$ for any $\mathcal{A} \in \mathcal{V}$ and any principal congruence $\theta$ on $\mathcal{A}$. A connective $f\left(p_{1}, \ldots, p_{n}\right)$ is called $t d$-distributive if $t d\left(p, q, f\left(r_{1}, \ldots, r_{n}\right)\right) \approx f\left(t d\left(p, q, r_{1}\right), \ldots, t d\left(p, q, r_{n}\right)\right)$. If $\mathrm{L}$ is a propositional logic and $\mathcal{V}$ is a corresponding variety (algebraic semantic) that has a $\mathrm{TD}$ term $t d$, then any admissible in $\mathrm{L}$ rule, the premises of which contain only $t d$-distributive operations, is derivable, and the substitution $r \mapsto t d(p, q, r)$ is a projective L-unifier for any formula containing only $t d$-distributive connectives. The above substitution is a generalization of the substitution introduced by T. Prucnal to prove structural completeness of the implication fragment of intuitionistic propositional logic.
\end{abstract}

\section{Introduction}

In this paper, we study admissibility of structural inference rules in algebraizable (propositional) logics. A (structural inference) rule is an expression of the form $A_{1}, \ldots, A_{n} / B$, where $A_{1}, \ldots, A_{n}$ are (propositional) formulas called premises (of the rule) and $B$ is a formula called the conclusion (of the rule). We recall that given a (propositional) logic $\mathrm{L}$, a rule $A_{1}, \ldots, A_{n} / B$ is admissible in $\mathrm{L}$ if the logic $\mathrm{L}$ is closed under this rule; that is, for any substitution $\sigma$ (of formulas for propositional variables), formula $\sigma(B)$ is valid in $L$ as long as all formulas $\sigma\left(A_{1}\right), \ldots, \sigma\left(A_{n}\right)$ are valid in $\mathrm{L}$. A substitution that simultaneously makes all formulas $A_{1}, \ldots, A_{n}$ valid in $\mathrm{L}$ is known as an L-unifier of formulas $A_{1}, \ldots, A_{n}$. An L-unifier of a single formula is a substitution that makes this formula valid in $L$. Thus, a rule is admissible in a

Received September 30, 2012; accepted January 30, 2014

First published online August 13, 2016

2010 Mathematics Subject Classification: Primary 03B55, 03B60; Secondary 03G27, 03G25

Keywords: propositional logic, admissible inference rule, unification, hereditary structural completeness, algebraic semantic, Prucnal's substitution, ternary deduction term

(C) 2016 by University of Notre Dame 10.1215/00294527-3659423 


\title{
Algebraic Logic Perspective on Prucnal's Substitution
}

\author{
Alex Citkin
}

\begin{abstract}
A term $\operatorname{td}(p, q, r)$ is called a ternary deductive (TD) term for a variety of algebras $\mathcal{V}$ if the identity $t d(p, p, r) \approx r$ holds in $\mathcal{V}$ and $(\mathrm{c}, \mathrm{d}) \in \theta(\mathrm{a}, \mathrm{b})$ yields $t d(\mathrm{a}, \mathrm{b}, \mathrm{c}) \approx t d(\mathrm{a}, \mathrm{b}, \mathrm{d})$ for any $\mathcal{A} \in \mathcal{V}$ and any principal congruence $\theta$ on $\mathcal{A}$. A connective $f\left(p_{1}, \ldots, p_{n}\right)$ is called $t d$-distributive if $t d\left(p, q, f\left(r_{1}, \ldots, r_{n}\right)\right) \approx f\left(t d\left(p, q, r_{1}\right), \ldots, t d\left(p, q, r_{n}\right)\right)$. If $\mathrm{L}$ is a propositional logic and $\mathcal{V}$ is a corresponding variety (algebraic semantic) that has a $\mathrm{TD}$ term $t d$, then any admissible in $\mathrm{L}$ rule, the premises of which contain only $t d$-distributive operations, is derivable, and the substitution $r \mapsto t d(p, q, r)$ is a projective L-unifier for any formula containing only $t d$-distributive connectives. The above substitution is a generalization of the substitution introduced by T. Prucnal to prove structural completeness of the implication fragment of intuitionistic propositional logic.
\end{abstract}

\section{Introduction}

In this paper, we study admissibility of structural inference rules in algebraizable (propositional) logics. A (structural inference) rule is an expression of the form $A_{1}, \ldots, A_{n} / B$, where $A_{1}, \ldots, A_{n}$ are (propositional) formulas called premises (of the rule) and $B$ is a formula called the conclusion (of the rule). We recall that given a (propositional) logic $\mathrm{L}$, a rule $A_{1}, \ldots, A_{n} / B$ is admissible in $\mathrm{L}$ if the logic $\mathrm{L}$ is closed under this rule; that is, for any substitution $\sigma$ (of formulas for propositional variables), formula $\sigma(B)$ is valid in $L$ as long as all formulas $\sigma\left(A_{1}\right), \ldots, \sigma\left(A_{n}\right)$ are valid in $\mathrm{L}$. A substitution that simultaneously makes all formulas $A_{1}, \ldots, A_{n}$ valid in $\mathrm{L}$ is known as an L-unifier of formulas $A_{1}, \ldots, A_{n}$. An L-unifier of a single formula is a substitution that makes this formula valid in $L$. Thus, a rule is admissible in a

Received September 30, 2012; accepted January 30, 2014

First published online August 13, 2016

2010 Mathematics Subject Classification: Primary 03B55, 03B60; Secondary 03G27, 03G25

Keywords: propositional logic, admissible inference rule, unification, hereditary structural completeness, algebraic semantic, Prucnal's substitution, ternary deduction term

(C) 2016 by University of Notre Dame 10.1215/00294527-3659423 


\title{
Algebraic Logic Perspective on Prucnal's Substitution
}

\author{
Alex Citkin
}

\begin{abstract}
A term $\operatorname{td}(p, q, r)$ is called a ternary deductive (TD) term for a variety of algebras $\mathcal{V}$ if the identity $t d(p, p, r) \approx r$ holds in $\mathcal{V}$ and $(\mathrm{c}, \mathrm{d}) \in \theta(\mathrm{a}, \mathrm{b})$ yields $t d(\mathrm{a}, \mathrm{b}, \mathrm{c}) \approx t d(\mathrm{a}, \mathrm{b}, \mathrm{d})$ for any $\mathcal{A} \in \mathcal{V}$ and any principal congruence $\theta$ on $\mathcal{A}$. A connective $f\left(p_{1}, \ldots, p_{n}\right)$ is called $t d$-distributive if $t d\left(p, q, f\left(r_{1}, \ldots, r_{n}\right)\right) \approx f\left(t d\left(p, q, r_{1}\right), \ldots, t d\left(p, q, r_{n}\right)\right)$. If $\mathrm{L}$ is a propositional logic and $\mathcal{V}$ is a corresponding variety (algebraic semantic) that has a $\mathrm{TD}$ term $t d$, then any admissible in $\mathrm{L}$ rule, the premises of which contain only $t d$-distributive operations, is derivable, and the substitution $r \mapsto t d(p, q, r)$ is a projective L-unifier for any formula containing only $t d$-distributive connectives. The above substitution is a generalization of the substitution introduced by T. Prucnal to prove structural completeness of the implication fragment of intuitionistic propositional logic.
\end{abstract}

\section{Introduction}

In this paper, we study admissibility of structural inference rules in algebraizable (propositional) logics. A (structural inference) rule is an expression of the form $A_{1}, \ldots, A_{n} / B$, where $A_{1}, \ldots, A_{n}$ are (propositional) formulas called premises (of the rule) and $B$ is a formula called the conclusion (of the rule). We recall that given a (propositional) logic $\mathrm{L}$, a rule $A_{1}, \ldots, A_{n} / B$ is admissible in $\mathrm{L}$ if the logic $\mathrm{L}$ is closed under this rule; that is, for any substitution $\sigma$ (of formulas for propositional variables), formula $\sigma(B)$ is valid in $L$ as long as all formulas $\sigma\left(A_{1}\right), \ldots, \sigma\left(A_{n}\right)$ are valid in $\mathrm{L}$. A substitution that simultaneously makes all formulas $A_{1}, \ldots, A_{n}$ valid in $\mathrm{L}$ is known as an L-unifier of formulas $A_{1}, \ldots, A_{n}$. An L-unifier of a single formula is a substitution that makes this formula valid in $L$. Thus, a rule is admissible in a

Received September 30, 2012; accepted January 30, 2014

First published online August 13, 2016

2010 Mathematics Subject Classification: Primary 03B55, 03B60; Secondary 03G27, 03G25

Keywords: propositional logic, admissible inference rule, unification, hereditary structural completeness, algebraic semantic, Prucnal's substitution, ternary deduction term

(C) 2016 by University of Notre Dame 10.1215/00294527-3659423 


\title{
Algebraic Logic Perspective on Prucnal's Substitution
}

\author{
Alex Citkin
}

\begin{abstract}
A term $\operatorname{td}(p, q, r)$ is called a ternary deductive (TD) term for a variety of algebras $\mathcal{V}$ if the identity $t d(p, p, r) \approx r$ holds in $\mathcal{V}$ and $(\mathrm{c}, \mathrm{d}) \in \theta(\mathrm{a}, \mathrm{b})$ yields $t d(\mathrm{a}, \mathrm{b}, \mathrm{c}) \approx t d(\mathrm{a}, \mathrm{b}, \mathrm{d})$ for any $\mathcal{A} \in \mathcal{V}$ and any principal congruence $\theta$ on $\mathcal{A}$. A connective $f\left(p_{1}, \ldots, p_{n}\right)$ is called $t d$-distributive if $t d\left(p, q, f\left(r_{1}, \ldots, r_{n}\right)\right) \approx f\left(t d\left(p, q, r_{1}\right), \ldots, t d\left(p, q, r_{n}\right)\right)$. If $\mathrm{L}$ is a propositional logic and $\mathcal{V}$ is a corresponding variety (algebraic semantic) that has a $\mathrm{TD}$ term $t d$, then any admissible in $\mathrm{L}$ rule, the premises of which contain only $t d$-distributive operations, is derivable, and the substitution $r \mapsto t d(p, q, r)$ is a projective L-unifier for any formula containing only $t d$-distributive connectives. The above substitution is a generalization of the substitution introduced by T. Prucnal to prove structural completeness of the implication fragment of intuitionistic propositional logic.
\end{abstract}

\section{Introduction}

In this paper, we study admissibility of structural inference rules in algebraizable (propositional) logics. A (structural inference) rule is an expression of the form $A_{1}, \ldots, A_{n} / B$, where $A_{1}, \ldots, A_{n}$ are (propositional) formulas called premises (of the rule) and $B$ is a formula called the conclusion (of the rule). We recall that given a (propositional) logic $\mathrm{L}$, a rule $A_{1}, \ldots, A_{n} / B$ is admissible in $\mathrm{L}$ if the logic $\mathrm{L}$ is closed under this rule; that is, for any substitution $\sigma$ (of formulas for propositional variables), formula $\sigma(B)$ is valid in $L$ as long as all formulas $\sigma\left(A_{1}\right), \ldots, \sigma\left(A_{n}\right)$ are valid in $\mathrm{L}$. A substitution that simultaneously makes all formulas $A_{1}, \ldots, A_{n}$ valid in $\mathrm{L}$ is known as an L-unifier of formulas $A_{1}, \ldots, A_{n}$. An L-unifier of a single formula is a substitution that makes this formula valid in $L$. Thus, a rule is admissible in a

Received September 30, 2012; accepted January 30, 2014

First published online August 13, 2016

2010 Mathematics Subject Classification: Primary 03B55, 03B60; Secondary 03G27, 03G25

Keywords: propositional logic, admissible inference rule, unification, hereditary structural completeness, algebraic semantic, Prucnal's substitution, ternary deduction term

(C) 2016 by University of Notre Dame 10.1215/00294527-3659423 


\title{
Algebraic Logic Perspective on Prucnal's Substitution
}

\author{
Alex Citkin
}

\begin{abstract}
A term $\operatorname{td}(p, q, r)$ is called a ternary deductive (TD) term for a variety of algebras $\mathcal{V}$ if the identity $t d(p, p, r) \approx r$ holds in $\mathcal{V}$ and $(\mathrm{c}, \mathrm{d}) \in \theta(\mathrm{a}, \mathrm{b})$ yields $t d(\mathrm{a}, \mathrm{b}, \mathrm{c}) \approx t d(\mathrm{a}, \mathrm{b}, \mathrm{d})$ for any $\mathcal{A} \in \mathcal{V}$ and any principal congruence $\theta$ on $\mathcal{A}$. A connective $f\left(p_{1}, \ldots, p_{n}\right)$ is called $t d$-distributive if $t d\left(p, q, f\left(r_{1}, \ldots, r_{n}\right)\right) \approx f\left(t d\left(p, q, r_{1}\right), \ldots, t d\left(p, q, r_{n}\right)\right)$. If $\mathrm{L}$ is a propositional logic and $\mathcal{V}$ is a corresponding variety (algebraic semantic) that has a $\mathrm{TD}$ term $t d$, then any admissible in $\mathrm{L}$ rule, the premises of which contain only $t d$-distributive operations, is derivable, and the substitution $r \mapsto t d(p, q, r)$ is a projective L-unifier for any formula containing only $t d$-distributive connectives. The above substitution is a generalization of the substitution introduced by T. Prucnal to prove structural completeness of the implication fragment of intuitionistic propositional logic.
\end{abstract}

\section{Introduction}

In this paper, we study admissibility of structural inference rules in algebraizable (propositional) logics. A (structural inference) rule is an expression of the form $A_{1}, \ldots, A_{n} / B$, where $A_{1}, \ldots, A_{n}$ are (propositional) formulas called premises (of the rule) and $B$ is a formula called the conclusion (of the rule). We recall that given a (propositional) logic $\mathrm{L}$, a rule $A_{1}, \ldots, A_{n} / B$ is admissible in $\mathrm{L}$ if the logic $\mathrm{L}$ is closed under this rule; that is, for any substitution $\sigma$ (of formulas for propositional variables), formula $\sigma(B)$ is valid in $L$ as long as all formulas $\sigma\left(A_{1}\right), \ldots, \sigma\left(A_{n}\right)$ are valid in $\mathrm{L}$. A substitution that simultaneously makes all formulas $A_{1}, \ldots, A_{n}$ valid in $\mathrm{L}$ is known as an L-unifier of formulas $A_{1}, \ldots, A_{n}$. An L-unifier of a single formula is a substitution that makes this formula valid in $L$. Thus, a rule is admissible in a

Received September 30, 2012; accepted January 30, 2014

First published online August 13, 2016

2010 Mathematics Subject Classification: Primary 03B55, 03B60; Secondary 03G27, 03G25

Keywords: propositional logic, admissible inference rule, unification, hereditary structural completeness, algebraic semantic, Prucnal's substitution, ternary deduction term

(C) 2016 by University of Notre Dame 10.1215/00294527-3659423 


\title{
Algebraic Logic Perspective on Prucnal's Substitution
}

\author{
Alex Citkin
}

\begin{abstract}
A term $\operatorname{td}(p, q, r)$ is called a ternary deductive (TD) term for a variety of algebras $\mathcal{V}$ if the identity $t d(p, p, r) \approx r$ holds in $\mathcal{V}$ and $(\mathrm{c}, \mathrm{d}) \in \theta(\mathrm{a}, \mathrm{b})$ yields $t d(\mathrm{a}, \mathrm{b}, \mathrm{c}) \approx t d(\mathrm{a}, \mathrm{b}, \mathrm{d})$ for any $\mathcal{A} \in \mathcal{V}$ and any principal congruence $\theta$ on $\mathcal{A}$. A connective $f\left(p_{1}, \ldots, p_{n}\right)$ is called $t d$-distributive if $t d\left(p, q, f\left(r_{1}, \ldots, r_{n}\right)\right) \approx f\left(t d\left(p, q, r_{1}\right), \ldots, t d\left(p, q, r_{n}\right)\right)$. If $\mathrm{L}$ is a propositional logic and $\mathcal{V}$ is a corresponding variety (algebraic semantic) that has a $\mathrm{TD}$ term $t d$, then any admissible in $\mathrm{L}$ rule, the premises of which contain only $t d$-distributive operations, is derivable, and the substitution $r \mapsto t d(p, q, r)$ is a projective L-unifier for any formula containing only $t d$-distributive connectives. The above substitution is a generalization of the substitution introduced by T. Prucnal to prove structural completeness of the implication fragment of intuitionistic propositional logic.
\end{abstract}

\section{Introduction}

In this paper, we study admissibility of structural inference rules in algebraizable (propositional) logics. A (structural inference) rule is an expression of the form $A_{1}, \ldots, A_{n} / B$, where $A_{1}, \ldots, A_{n}$ are (propositional) formulas called premises (of the rule) and $B$ is a formula called the conclusion (of the rule). We recall that given a (propositional) logic $\mathrm{L}$, a rule $A_{1}, \ldots, A_{n} / B$ is admissible in $\mathrm{L}$ if the logic $\mathrm{L}$ is closed under this rule; that is, for any substitution $\sigma$ (of formulas for propositional variables), formula $\sigma(B)$ is valid in $L$ as long as all formulas $\sigma\left(A_{1}\right), \ldots, \sigma\left(A_{n}\right)$ are valid in $\mathrm{L}$. A substitution that simultaneously makes all formulas $A_{1}, \ldots, A_{n}$ valid in $\mathrm{L}$ is known as an L-unifier of formulas $A_{1}, \ldots, A_{n}$. An L-unifier of a single formula is a substitution that makes this formula valid in $L$. Thus, a rule is admissible in a

Received September 30, 2012; accepted January 30, 2014

First published online August 13, 2016

2010 Mathematics Subject Classification: Primary 03B55, 03B60; Secondary 03G27, 03G25

Keywords: propositional logic, admissible inference rule, unification, hereditary structural completeness, algebraic semantic, Prucnal's substitution, ternary deduction term

(C) 2016 by University of Notre Dame 10.1215/00294527-3659423 


\title{
Algebraic Logic Perspective on Prucnal's Substitution
}

\author{
Alex Citkin
}

\begin{abstract}
A term $\operatorname{td}(p, q, r)$ is called a ternary deductive (TD) term for a variety of algebras $\mathcal{V}$ if the identity $t d(p, p, r) \approx r$ holds in $\mathcal{V}$ and $(\mathrm{c}, \mathrm{d}) \in \theta(\mathrm{a}, \mathrm{b})$ yields $t d(\mathrm{a}, \mathrm{b}, \mathrm{c}) \approx t d(\mathrm{a}, \mathrm{b}, \mathrm{d})$ for any $\mathcal{A} \in \mathcal{V}$ and any principal congruence $\theta$ on $\mathcal{A}$. A connective $f\left(p_{1}, \ldots, p_{n}\right)$ is called $t d$-distributive if $t d\left(p, q, f\left(r_{1}, \ldots, r_{n}\right)\right) \approx f\left(t d\left(p, q, r_{1}\right), \ldots, t d\left(p, q, r_{n}\right)\right)$. If $\mathrm{L}$ is a propositional logic and $\mathcal{V}$ is a corresponding variety (algebraic semantic) that has a $\mathrm{TD}$ term $t d$, then any admissible in $\mathrm{L}$ rule, the premises of which contain only $t d$-distributive operations, is derivable, and the substitution $r \mapsto t d(p, q, r)$ is a projective L-unifier for any formula containing only $t d$-distributive connectives. The above substitution is a generalization of the substitution introduced by T. Prucnal to prove structural completeness of the implication fragment of intuitionistic propositional logic.
\end{abstract}

\section{Introduction}

In this paper, we study admissibility of structural inference rules in algebraizable (propositional) logics. A (structural inference) rule is an expression of the form $A_{1}, \ldots, A_{n} / B$, where $A_{1}, \ldots, A_{n}$ are (propositional) formulas called premises (of the rule) and $B$ is a formula called the conclusion (of the rule). We recall that given a (propositional) logic $\mathrm{L}$, a rule $A_{1}, \ldots, A_{n} / B$ is admissible in $\mathrm{L}$ if the logic $\mathrm{L}$ is closed under this rule; that is, for any substitution $\sigma$ (of formulas for propositional variables), formula $\sigma(B)$ is valid in $L$ as long as all formulas $\sigma\left(A_{1}\right), \ldots, \sigma\left(A_{n}\right)$ are valid in $\mathrm{L}$. A substitution that simultaneously makes all formulas $A_{1}, \ldots, A_{n}$ valid in $\mathrm{L}$ is known as an L-unifier of formulas $A_{1}, \ldots, A_{n}$. An L-unifier of a single formula is a substitution that makes this formula valid in $L$. Thus, a rule is admissible in a

Received September 30, 2012; accepted January 30, 2014

First published online August 13, 2016

2010 Mathematics Subject Classification: Primary 03B55, 03B60; Secondary 03G27, 03G25

Keywords: propositional logic, admissible inference rule, unification, hereditary structural completeness, algebraic semantic, Prucnal's substitution, ternary deduction term

(C) 2016 by University of Notre Dame 10.1215/00294527-3659423 


\title{
Algebraic Logic Perspective on Prucnal's Substitution
}

\author{
Alex Citkin
}

\begin{abstract}
A term $\operatorname{td}(p, q, r)$ is called a ternary deductive (TD) term for a variety of algebras $\mathcal{V}$ if the identity $t d(p, p, r) \approx r$ holds in $\mathcal{V}$ and $(\mathrm{c}, \mathrm{d}) \in \theta(\mathrm{a}, \mathrm{b})$ yields $t d(\mathrm{a}, \mathrm{b}, \mathrm{c}) \approx t d(\mathrm{a}, \mathrm{b}, \mathrm{d})$ for any $\mathcal{A} \in \mathcal{V}$ and any principal congruence $\theta$ on $\mathcal{A}$. A connective $f\left(p_{1}, \ldots, p_{n}\right)$ is called $t d$-distributive if $t d\left(p, q, f\left(r_{1}, \ldots, r_{n}\right)\right) \approx f\left(t d\left(p, q, r_{1}\right), \ldots, t d\left(p, q, r_{n}\right)\right)$. If $\mathrm{L}$ is a propositional logic and $\mathcal{V}$ is a corresponding variety (algebraic semantic) that has a $\mathrm{TD}$ term $t d$, then any admissible in $\mathrm{L}$ rule, the premises of which contain only $t d$-distributive operations, is derivable, and the substitution $r \mapsto t d(p, q, r)$ is a projective L-unifier for any formula containing only $t d$-distributive connectives. The above substitution is a generalization of the substitution introduced by T. Prucnal to prove structural completeness of the implication fragment of intuitionistic propositional logic.
\end{abstract}

\section{Introduction}

In this paper, we study admissibility of structural inference rules in algebraizable (propositional) logics. A (structural inference) rule is an expression of the form $A_{1}, \ldots, A_{n} / B$, where $A_{1}, \ldots, A_{n}$ are (propositional) formulas called premises (of the rule) and $B$ is a formula called the conclusion (of the rule). We recall that given a (propositional) logic $\mathrm{L}$, a rule $A_{1}, \ldots, A_{n} / B$ is admissible in $\mathrm{L}$ if the logic $\mathrm{L}$ is closed under this rule; that is, for any substitution $\sigma$ (of formulas for propositional variables), formula $\sigma(B)$ is valid in $L$ as long as all formulas $\sigma\left(A_{1}\right), \ldots, \sigma\left(A_{n}\right)$ are valid in $\mathrm{L}$. A substitution that simultaneously makes all formulas $A_{1}, \ldots, A_{n}$ valid in $\mathrm{L}$ is known as an L-unifier of formulas $A_{1}, \ldots, A_{n}$. An L-unifier of a single formula is a substitution that makes this formula valid in $L$. Thus, a rule is admissible in a

Received September 30, 2012; accepted January 30, 2014

First published online August 13, 2016

2010 Mathematics Subject Classification: Primary 03B55, 03B60; Secondary 03G27, 03G25

Keywords: propositional logic, admissible inference rule, unification, hereditary structural completeness, algebraic semantic, Prucnal's substitution, ternary deduction term

(C) 2016 by University of Notre Dame 10.1215/00294527-3659423 


\title{
Algebraic Logic Perspective on Prucnal's Substitution
}

\author{
Alex Citkin
}

\begin{abstract}
A term $\operatorname{td}(p, q, r)$ is called a ternary deductive (TD) term for a variety of algebras $\mathcal{V}$ if the identity $t d(p, p, r) \approx r$ holds in $\mathcal{V}$ and $(\mathrm{c}, \mathrm{d}) \in \theta(\mathrm{a}, \mathrm{b})$ yields $t d(\mathrm{a}, \mathrm{b}, \mathrm{c}) \approx t d(\mathrm{a}, \mathrm{b}, \mathrm{d})$ for any $\mathcal{A} \in \mathcal{V}$ and any principal congruence $\theta$ on $\mathcal{A}$. A connective $f\left(p_{1}, \ldots, p_{n}\right)$ is called $t d$-distributive if $t d\left(p, q, f\left(r_{1}, \ldots, r_{n}\right)\right) \approx f\left(t d\left(p, q, r_{1}\right), \ldots, t d\left(p, q, r_{n}\right)\right)$. If $\mathrm{L}$ is a propositional logic and $\mathcal{V}$ is a corresponding variety (algebraic semantic) that has a $\mathrm{TD}$ term $t d$, then any admissible in $\mathrm{L}$ rule, the premises of which contain only $t d$-distributive operations, is derivable, and the substitution $r \mapsto t d(p, q, r)$ is a projective L-unifier for any formula containing only $t d$-distributive connectives. The above substitution is a generalization of the substitution introduced by T. Prucnal to prove structural completeness of the implication fragment of intuitionistic propositional logic.
\end{abstract}

\section{Introduction}

In this paper, we study admissibility of structural inference rules in algebraizable (propositional) logics. A (structural inference) rule is an expression of the form $A_{1}, \ldots, A_{n} / B$, where $A_{1}, \ldots, A_{n}$ are (propositional) formulas called premises (of the rule) and $B$ is a formula called the conclusion (of the rule). We recall that given a (propositional) logic $\mathrm{L}$, a rule $A_{1}, \ldots, A_{n} / B$ is admissible in $\mathrm{L}$ if the logic $\mathrm{L}$ is closed under this rule; that is, for any substitution $\sigma$ (of formulas for propositional variables), formula $\sigma(B)$ is valid in $L$ as long as all formulas $\sigma\left(A_{1}\right), \ldots, \sigma\left(A_{n}\right)$ are valid in $\mathrm{L}$. A substitution that simultaneously makes all formulas $A_{1}, \ldots, A_{n}$ valid in $\mathrm{L}$ is known as an L-unifier of formulas $A_{1}, \ldots, A_{n}$. An L-unifier of a single formula is a substitution that makes this formula valid in $L$. Thus, a rule is admissible in a

Received September 30, 2012; accepted January 30, 2014

First published online August 13, 2016

2010 Mathematics Subject Classification: Primary 03B55, 03B60; Secondary 03G27, 03G25

Keywords: propositional logic, admissible inference rule, unification, hereditary structural completeness, algebraic semantic, Prucnal's substitution, ternary deduction term

(C) 2016 by University of Notre Dame 10.1215/00294527-3659423 


\title{
Algebraic Logic Perspective on Prucnal's Substitution
}

\author{
Alex Citkin
}

\begin{abstract}
A term $\operatorname{td}(p, q, r)$ is called a ternary deductive (TD) term for a variety of algebras $\mathcal{V}$ if the identity $t d(p, p, r) \approx r$ holds in $\mathcal{V}$ and $(\mathrm{c}, \mathrm{d}) \in \theta(\mathrm{a}, \mathrm{b})$ yields $t d(\mathrm{a}, \mathrm{b}, \mathrm{c}) \approx t d(\mathrm{a}, \mathrm{b}, \mathrm{d})$ for any $\mathcal{A} \in \mathcal{V}$ and any principal congruence $\theta$ on $\mathcal{A}$. A connective $f\left(p_{1}, \ldots, p_{n}\right)$ is called $t d$-distributive if $t d\left(p, q, f\left(r_{1}, \ldots, r_{n}\right)\right) \approx f\left(t d\left(p, q, r_{1}\right), \ldots, t d\left(p, q, r_{n}\right)\right)$. If $\mathrm{L}$ is a propositional logic and $\mathcal{V}$ is a corresponding variety (algebraic semantic) that has a $\mathrm{TD}$ term $t d$, then any admissible in $\mathrm{L}$ rule, the premises of which contain only $t d$-distributive operations, is derivable, and the substitution $r \mapsto t d(p, q, r)$ is a projective L-unifier for any formula containing only $t d$-distributive connectives. The above substitution is a generalization of the substitution introduced by T. Prucnal to prove structural completeness of the implication fragment of intuitionistic propositional logic.
\end{abstract}

\section{Introduction}

In this paper, we study admissibility of structural inference rules in algebraizable (propositional) logics. A (structural inference) rule is an expression of the form $A_{1}, \ldots, A_{n} / B$, where $A_{1}, \ldots, A_{n}$ are (propositional) formulas called premises (of the rule) and $B$ is a formula called the conclusion (of the rule). We recall that given a (propositional) logic $\mathrm{L}$, a rule $A_{1}, \ldots, A_{n} / B$ is admissible in $\mathrm{L}$ if the logic $\mathrm{L}$ is closed under this rule; that is, for any substitution $\sigma$ (of formulas for propositional variables), formula $\sigma(B)$ is valid in $L$ as long as all formulas $\sigma\left(A_{1}\right), \ldots, \sigma\left(A_{n}\right)$ are valid in $\mathrm{L}$. A substitution that simultaneously makes all formulas $A_{1}, \ldots, A_{n}$ valid in $\mathrm{L}$ is known as an L-unifier of formulas $A_{1}, \ldots, A_{n}$. An L-unifier of a single formula is a substitution that makes this formula valid in $L$. Thus, a rule is admissible in a

Received September 30, 2012; accepted January 30, 2014

First published online August 13, 2016

2010 Mathematics Subject Classification: Primary 03B55, 03B60; Secondary 03G27, 03G25

Keywords: propositional logic, admissible inference rule, unification, hereditary structural completeness, algebraic semantic, Prucnal's substitution, ternary deduction term

(C) 2016 by University of Notre Dame 10.1215/00294527-3659423 


\title{
Algebraic Logic Perspective on Prucnal's Substitution
}

\author{
Alex Citkin
}

\begin{abstract}
A term $\operatorname{td}(p, q, r)$ is called a ternary deductive (TD) term for a variety of algebras $\mathcal{V}$ if the identity $t d(p, p, r) \approx r$ holds in $\mathcal{V}$ and $(\mathrm{c}, \mathrm{d}) \in \theta(\mathrm{a}, \mathrm{b})$ yields $t d(\mathrm{a}, \mathrm{b}, \mathrm{c}) \approx t d(\mathrm{a}, \mathrm{b}, \mathrm{d})$ for any $\mathcal{A} \in \mathcal{V}$ and any principal congruence $\theta$ on $\mathcal{A}$. A connective $f\left(p_{1}, \ldots, p_{n}\right)$ is called $t d$-distributive if $t d\left(p, q, f\left(r_{1}, \ldots, r_{n}\right)\right) \approx f\left(t d\left(p, q, r_{1}\right), \ldots, t d\left(p, q, r_{n}\right)\right)$. If $\mathrm{L}$ is a propositional logic and $\mathcal{V}$ is a corresponding variety (algebraic semantic) that has a $\mathrm{TD}$ term $t d$, then any admissible in $\mathrm{L}$ rule, the premises of which contain only $t d$-distributive operations, is derivable, and the substitution $r \mapsto t d(p, q, r)$ is a projective L-unifier for any formula containing only $t d$-distributive connectives. The above substitution is a generalization of the substitution introduced by T. Prucnal to prove structural completeness of the implication fragment of intuitionistic propositional logic.
\end{abstract}

\section{Introduction}

In this paper, we study admissibility of structural inference rules in algebraizable (propositional) logics. A (structural inference) rule is an expression of the form $A_{1}, \ldots, A_{n} / B$, where $A_{1}, \ldots, A_{n}$ are (propositional) formulas called premises (of the rule) and $B$ is a formula called the conclusion (of the rule). We recall that given a (propositional) logic $\mathrm{L}$, a rule $A_{1}, \ldots, A_{n} / B$ is admissible in $\mathrm{L}$ if the logic $\mathrm{L}$ is closed under this rule; that is, for any substitution $\sigma$ (of formulas for propositional variables), formula $\sigma(B)$ is valid in $L$ as long as all formulas $\sigma\left(A_{1}\right), \ldots, \sigma\left(A_{n}\right)$ are valid in $\mathrm{L}$. A substitution that simultaneously makes all formulas $A_{1}, \ldots, A_{n}$ valid in $\mathrm{L}$ is known as an L-unifier of formulas $A_{1}, \ldots, A_{n}$. An L-unifier of a single formula is a substitution that makes this formula valid in $L$. Thus, a rule is admissible in a

Received September 30, 2012; accepted January 30, 2014

First published online August 13, 2016

2010 Mathematics Subject Classification: Primary 03B55, 03B60; Secondary 03G27, 03G25

Keywords: propositional logic, admissible inference rule, unification, hereditary structural completeness, algebraic semantic, Prucnal's substitution, ternary deduction term

(C) 2016 by University of Notre Dame 10.1215/00294527-3659423 


\title{
Algebraic Logic Perspective on Prucnal's Substitution
}

\author{
Alex Citkin
}

\begin{abstract}
A term $\operatorname{td}(p, q, r)$ is called a ternary deductive (TD) term for a variety of algebras $\mathcal{V}$ if the identity $t d(p, p, r) \approx r$ holds in $\mathcal{V}$ and $(\mathrm{c}, \mathrm{d}) \in \theta(\mathrm{a}, \mathrm{b})$ yields $t d(\mathrm{a}, \mathrm{b}, \mathrm{c}) \approx t d(\mathrm{a}, \mathrm{b}, \mathrm{d})$ for any $\mathcal{A} \in \mathcal{V}$ and any principal congruence $\theta$ on $\mathcal{A}$. A connective $f\left(p_{1}, \ldots, p_{n}\right)$ is called $t d$-distributive if $t d\left(p, q, f\left(r_{1}, \ldots, r_{n}\right)\right) \approx f\left(t d\left(p, q, r_{1}\right), \ldots, t d\left(p, q, r_{n}\right)\right)$. If $\mathrm{L}$ is a propositional logic and $\mathcal{V}$ is a corresponding variety (algebraic semantic) that has a $\mathrm{TD}$ term $t d$, then any admissible in $\mathrm{L}$ rule, the premises of which contain only $t d$-distributive operations, is derivable, and the substitution $r \mapsto t d(p, q, r)$ is a projective L-unifier for any formula containing only $t d$-distributive connectives. The above substitution is a generalization of the substitution introduced by T. Prucnal to prove structural completeness of the implication fragment of intuitionistic propositional logic.
\end{abstract}

\section{Introduction}

In this paper, we study admissibility of structural inference rules in algebraizable (propositional) logics. A (structural inference) rule is an expression of the form $A_{1}, \ldots, A_{n} / B$, where $A_{1}, \ldots, A_{n}$ are (propositional) formulas called premises (of the rule) and $B$ is a formula called the conclusion (of the rule). We recall that given a (propositional) logic $\mathrm{L}$, a rule $A_{1}, \ldots, A_{n} / B$ is admissible in $\mathrm{L}$ if the logic $\mathrm{L}$ is closed under this rule; that is, for any substitution $\sigma$ (of formulas for propositional variables), formula $\sigma(B)$ is valid in $L$ as long as all formulas $\sigma\left(A_{1}\right), \ldots, \sigma\left(A_{n}\right)$ are valid in $\mathrm{L}$. A substitution that simultaneously makes all formulas $A_{1}, \ldots, A_{n}$ valid in $\mathrm{L}$ is known as an L-unifier of formulas $A_{1}, \ldots, A_{n}$. An L-unifier of a single formula is a substitution that makes this formula valid in $L$. Thus, a rule is admissible in a

Received September 30, 2012; accepted January 30, 2014

First published online August 13, 2016

2010 Mathematics Subject Classification: Primary 03B55, 03B60; Secondary 03G27, 03G25

Keywords: propositional logic, admissible inference rule, unification, hereditary structural completeness, algebraic semantic, Prucnal's substitution, ternary deduction term

(C) 2016 by University of Notre Dame 10.1215/00294527-3659423 


\title{
Algebraic Logic Perspective on Prucnal's Substitution
}

\author{
Alex Citkin
}

\begin{abstract}
A term $\operatorname{td}(p, q, r)$ is called a ternary deductive (TD) term for a variety of algebras $\mathcal{V}$ if the identity $t d(p, p, r) \approx r$ holds in $\mathcal{V}$ and $(\mathrm{c}, \mathrm{d}) \in \theta(\mathrm{a}, \mathrm{b})$ yields $t d(\mathrm{a}, \mathrm{b}, \mathrm{c}) \approx t d(\mathrm{a}, \mathrm{b}, \mathrm{d})$ for any $\mathcal{A} \in \mathcal{V}$ and any principal congruence $\theta$ on $\mathcal{A}$. A connective $f\left(p_{1}, \ldots, p_{n}\right)$ is called $t d$-distributive if $t d\left(p, q, f\left(r_{1}, \ldots, r_{n}\right)\right) \approx f\left(t d\left(p, q, r_{1}\right), \ldots, t d\left(p, q, r_{n}\right)\right)$. If $\mathrm{L}$ is a propositional logic and $\mathcal{V}$ is a corresponding variety (algebraic semantic) that has a $\mathrm{TD}$ term $t d$, then any admissible in $\mathrm{L}$ rule, the premises of which contain only $t d$-distributive operations, is derivable, and the substitution $r \mapsto t d(p, q, r)$ is a projective L-unifier for any formula containing only $t d$-distributive connectives. The above substitution is a generalization of the substitution introduced by T. Prucnal to prove structural completeness of the implication fragment of intuitionistic propositional logic.
\end{abstract}

\section{Introduction}

In this paper, we study admissibility of structural inference rules in algebraizable (propositional) logics. A (structural inference) rule is an expression of the form $A_{1}, \ldots, A_{n} / B$, where $A_{1}, \ldots, A_{n}$ are (propositional) formulas called premises (of the rule) and $B$ is a formula called the conclusion (of the rule). We recall that given a (propositional) logic $\mathrm{L}$, a rule $A_{1}, \ldots, A_{n} / B$ is admissible in $\mathrm{L}$ if the logic $\mathrm{L}$ is closed under this rule; that is, for any substitution $\sigma$ (of formulas for propositional variables), formula $\sigma(B)$ is valid in $L$ as long as all formulas $\sigma\left(A_{1}\right), \ldots, \sigma\left(A_{n}\right)$ are valid in $\mathrm{L}$. A substitution that simultaneously makes all formulas $A_{1}, \ldots, A_{n}$ valid in $\mathrm{L}$ is known as an L-unifier of formulas $A_{1}, \ldots, A_{n}$. An L-unifier of a single formula is a substitution that makes this formula valid in $L$. Thus, a rule is admissible in a

Received September 30, 2012; accepted January 30, 2014

First published online August 13, 2016

2010 Mathematics Subject Classification: Primary 03B55, 03B60; Secondary 03G27, 03G25

Keywords: propositional logic, admissible inference rule, unification, hereditary structural completeness, algebraic semantic, Prucnal's substitution, ternary deduction term

(C) 2016 by University of Notre Dame 10.1215/00294527-3659423 